\title{
Ascent and descent of going-down rings for integral extensions
}

\section{David E. Dobbs}

The author and Ira J. Papick have termed an integral domain $R$ a going-down ring if $R \subset T$ satisfies going-down for each domain $T$ containing $R$. The present paper investigates conditions which, for an integral extension $A \subset B$ of domains, imply that $A$ (respectively $B$ ) is going-down whenever $B$ (respectively $A$ ) is going-down. This explains the "descent" (respectively "ascent") in the title. Two typical results (the first about descent, the second about ascent) are given next.

THEOREM. Let $R \subset T$ be an integral extension of domains such that $T$ is going-down. If either $T$ is quasi-local or $R$ is integrally closed, then $R$ is going-down.

THEOREM. Let $R$ be an integrally closed domain with quotient field $K$, and let $T$ be the integral closure of $R$ in an algebraic field extension $L$ of $K$. If the group of $K$-algebra automorphisms of the normal closure of $L / K$ is finite of order $2^{a} 3^{b}$ and if $R$ is going-down, then $T$ is going-down.

\section{Introduction}

Recall from [6] and [7] that a (commutative integral) domain $R$ is called a going-down ring in case $R \subset T$ satisfies going-down for each domain $T$ containing $R$. Examples of going-down rings are Prüfer domains, arbitrary domains of Krull dimension 1 , and the rings

Received 4 May 1976. This research was supported by a grant from the National Science Foundation of the USA. 
constructed by iterated restrained power series in [6, Corollary 4.4]. In this paper we investigate conditions which, for an integral extension $A \subset B$ of domains, imply that $A$ (respectively $B$ ) is going-down whenever $B$ (respectively $A$ ) is going-down; that is, conditions guaranteeing descent (respectively ascent) of going-down rings for integral extensions. An example of Heinzer and Ohm [10], summarized below in Section 2, shows that, without further assumptions, both descent and ascent fail. The extra conditions assumed in our results avoid certain features of the Heinzer-Ohm example and, in the case of the second part of Theorem 2.4 and Theorem 3.3, are motivated by the descent [9, Corollary 3] and ascent [17, p. 31 ] results for Prüfer domains.

A word about the propriety of motivating questions about going-down by results about Prüfer domains seems to be in order. The characterization of going-down rings in [7, Theorem 1] was in fact motivated by characterizations of Prüfer domains involving flatness (cf. [18, Theorem 4] and [6, Proposition 3.1]). Going-down has figured in the characterization of several kinds of Prüfer domains (cf. [4, Corollary 4.3], [5, Corollaries 4 and 10], [6, Propositions 2.5 and 2.7]). Moreover, the class of Prüfer domains and the class of going-down rings are each closed under localization, and both fail to be closed under adjunction of indeterminates.

Our descent results are given in Section 2. With the aid of a recent result of Brase [1], Corollary 2.5 leads to new examples of going-down rings. Ascent, which is surprisingly elusive, is studied in Section 3, whose main result depends on Theorem 2.4 about descent and on the structure of the ambient Galois group.

$\bar{D}$ will denote the integral closure of a domain $D$. Unexplained terminology is standard, as in [8] and [11].

\section{Descent}

Recall from [6] that a commutative ring $A$ is said to be treed in case $\operatorname{spec}(A)$, as a poset under inclusion, is a tree. By [6, Theorem 2.2], any going-down ring is treed. Another proof follows easily from [6, Proposition 3.2, (i) $\Longleftrightarrow$ (iii)]. By using the ideas surrounding [13, Theorem 2.5], Lewis has recently constructed a treed domain of Krull 
dimension 2 which is not a going-down ring. A related construction of Heinzer and Ohm [10, p. 6], originally developed for other reasons ( $c f$. [10, Example 2.2] and [2, p. 25]), will be summarized next in order to indicate what sorts of results about descent and ascent may be possible.

EXAMPLE 2.1 (Heinzer-Ohm). Let $K=k(y, z)$ where $y, z$ are indeterminates over an algebraically closed field $k$ of characteristic zero. A rank 2 valuation of $K$ over $k$ may be given by sending $y$ and $z$ to $(0,1)$ and $(1,0)$ respectively, and then taking the value of a polynomial in $k[y, z]$ to be the infimum of the values of its constituent monomials; let $V$ be the corresponding valuation ring. Let $V^{*}$ be the integral closure of $V$ in an algebraic closure of $K$. Set $D=k+J$, where $J$ is the Jacobson radical of $V^{*}$.

It is shown in [10] that $D$ is a quasilocal G-domain (in the sense of [11]) with infinitely many primes of height 1 . Thus $D$ is not treed and, in particular, $D$ is not going-down. Of course, $V^{*}$ is a Prüfer domain by Prüfer's ascent result, so that both $V$ and $V^{*}$ are going-down. As $D \subset V^{*}$ and $V \subset D$ are each integral, it follows that descent and ascent both fail in general.

In order to motivate the hypotheses selected for our results, we next isolate certain salient features of the preceding data. First, $D \subset V^{*}$ does not satisfy going-down: this suggests Corollary 2.3 below. Second, $V^{*}$ is not a valuation ring (that is $V^{*}$ is not quasilocal; of. Corollary 2.5 and the first part of Theorem 2.4). Third, $D$ is not integrally closed (cf. Theorem 3.3, Proposition 3.4, Corollary 3.5, and the second part of Theorem 2.4). Moreover, applying the construction in [8, Appendix 2] to $V \subset D$ shows, by [7, Corollary], that an integral overring of a (quasi-local) going-down ring need not itself be going-down ( $c f$. Proposition 3.4).

The next lemma will lead to our descent results. We use "unibranched" in the sense of [4].

LEMMA 2.2. Let $A \subset B$ be an integral extension of commutative rings, with $B$ treed. If each maximal ideal of $A$ is unibranched in $B$, then $A$ is unibranched in $B$ and $A \subset B$ satisfies going-down.

Proot. Let $Q_{1}$ and $Q_{2}$ be prime ideals of $B$ contracting to a 
prime $P$ of $A$. Select a maximal ideal $M$ of $A$ containing $P$. Since $A \subset B$ satisfies going-up, there are primes $N_{i}$ of $B$ such that $Q_{i} \subset N_{i}$ and $N_{i} \cap A=M \quad(i=1,2)$; the unibranchedness hypothesis gives $N_{1}=N_{2}$. Then $Q_{1}$ and $Q_{2}$ are comparable, since $B$ is treed. As $A \subset B$ satisfies incomparability, it follows that $Q_{1}=Q_{2}$, so that $A \subset B$ is unibranched. An easy application of lying over and going-up now shows that $A \subset B$ satisfies going-down.

COROLLARY 2.3. Let $R$ be a domain such that $\bar{R}$ is going-down. If $R \subset \bar{R}$ satisfies going-down (for example, by the Zemma, if each maximal ideal of $R$ is unibranched in $\vec{R}$ ), then $R$ is going-down.

Proof. By [7, Theorem 1], it is enough to show that $R \subset V$ satisfies going-down for each valuation overring $V$ of $R$. Note that $\bar{R} \subset V$. As $R \subset \bar{R}$ and $\bar{R} \subset V$ each satisfy doing-down, transitivity completes the proof.

THEOREM 2.4. Let $R \subset T$ be an integral extension of domains, with $T$ being going-down. Assume that either $T$ is quasi-local or $R$ is integrally closed. Then $R$ is going-down.

Proof. As in the preceding proof, we need to show that $R \subset V$ satisfies going-down for each valuation overring $V$ of $R$. First, observe that $R \subset T$ satisfies going-down, by Lemma 2.2, if $T$ is quasilocal; by the going-down result of Krull [12], if $R$ is integrally closed. Next, $T \subset V T$ satisfies going-down, since $T$ is going-down, so that $R \subset V T$ satisfies going-down. However, $V \subset V T$ satisfies lying over since $V T$ is integral over $V$. An application of [14, Lemma 2 (2)] implies that $R \subset V$ satisfies going-down, as required.

COROLLARY 2.5. Let $R$ be a domain such that $\bar{R}$ is a valuation ring. Then each overring of $R$ (including $R$ itself) is going-down.

Proof. The integral closure of any overring of $R$ is an overring of $\bar{R}$ and, hence, is a valuation ring. It therefore suffices to show that $R$ is going-down. Apply either the first case of Theorem 2.4 or the case noted parenthetically in the statement of Corollary 2.3.

REMARKS 2.6. (a) The ploy of passing to $V T$ in the above proof of Theorem 2.4 was suggested by McAdam's proof of [14, Theorem 1]. A slightly 
different proof of Theorem 2.4 proceeds as follows. Let $R$ and $V$ be as before, $P \subset M$ be primes of $R$, and $N$ be a prime of $V$ contracting to $M$. Choose a valuation ring $W$ of the quotient field of $T$ such that

(i) $V \subset W$ and

(ii) $N^{\prime}$, the maximal ideal of $W$, contracts to $N$.

Since $T \subset W$, Lemma 2.2 or [12] supplies a prime $Q$ of $T$ which is contained in $N^{\prime} \cap T$ and contracts to $P$. Then, since $T$ is going-down, some prime $N^{\prime \prime}$ of $W$ lies within $N^{\prime}$ and contracts to $Q$. Then $N^{\prime \prime} \cap V$ is the required prime of $V$.

(b) It follows from [6, Corollary 4.4 (i), (iii)] that the converse of Corollary 2.5 (even replacing "valuation ring" by "Prüfer domain") is false. By extending the above techniques, Papick [16] has recently characterized the quasi-local $i$-domains (domains satisfying the hypotheses in Corollary 2.5); also see Proposition 3.4 below. Along different lines, Brase [1, Theorem] has characterized the quasi-local $i$-domains as the domains for which the set of valuation ideals is closed under intersection. By Corollary 2.5 and [6, Theorem 2.2], the set of prime ideals of any such domain is linearly ordered by inclusion; Brase directly observed the corresponding fact about the (larger) set of valuation ideals.

\section{Ascent}

Our main interest in this section is the following question, which is motivated by the ascent result of Prüfer $[17$, p. 31]. (For another proof of Prüfer's result, see [8, Theorem 18.3].)

QUESTION 3.0. Let $R$ be going-down and integrally closed, with quotient field $K$. If $T$ is the integral closure of $R$ in a field extension $L$ of $K$, must $T$ be going-down?

PROPOSITION 3.1. The answer to Question 3.0 is affirmative in general if it may be so answered in case $R$ is quasi-local and $L / K$ is finite Galois with simple Galois group.

Proor. (i) Assume that $L / K$ is finite Galois with simple Galois group. To test whether $T$ is going-down, take a domain $B$ containing $T$, primes $Q \subset N$ of $T$, and a prime $I$ of $B$ contracting to $N$. Let $M=N \cap R$. By the hypothesis, $T_{R \backslash M}$ (which is the integral closure of 
the quasi-local going-down ring $R_{M}$ in $L$ ) is going-down. Thus $I B R M$ contains a prime $J$ of $B_{R \backslash M}$ contracting to ${ }^{Q T}{ }_{R \backslash M}$, whence $J \cap B$ is contained in $I$ and contracts to $Q$. Thus $T \subset B$ satisfies going-down, to complete this case.

(ii) Assume that $L / K$ is finite Galois, with Galois group $G$. Induct on $|G|$, the order of $G$. Without loss of generality, $G$ is neither simple nor trivial. Let $F$ be the fixed field (in the sense of Galois theory) of a proper normal subgroup $H$ of $G$. Now $F / K$ is finite Galois, with Galois group isomorphic to $G / H$. As $|G / H|<|G|$, the induction hypothesis shows that $S$, the integral closure of $R$ in $F$, is going-down. However, $T$ is the integral closure of $S$ in $L$, and $L / F$ is finite Galois with Galois group $H$, so that the induction hypothesis applies again, and establishes this case.

(iii) Assume that $L / K$ is finite normal. Let $F$ be the set of elements of $L$ which are purely inseparable over $K$, and let $S$ be the integral closure of $R$ in $F$. Since $L / F$ is finite Galois (with Galois group equal to the set of $K$-algebra automorphisms of $L$ ), it suffices to prove that $S$ is going-down. This follows readily since $R \subset S$ is unibranched [8, Corollary 10.3] and $R$ is going-down.

(iv) The case of finite (algebraic) $L / K$ follows by passing to the normal closure of $L / K$ (to which (iii) applies) and then using an earlier descent result, the second part of Theorem 2.4 .

(v) General case. Deny. Then there exist primes $Q_{1} \subset N$ of $T$ and a prime $I$ of an overring $V$ of $T$ such that $I$ contracts to $N$ and $I$ contains no prime of $V$ contracting to $Q_{1}$. Since $R \subset V$ satisfies going-down, there is a prime $J$ of $V$ such that $J \subset I$ and $J \cap R=Q_{1} \cap R$. As $Q_{2}=J \cap T$ must be distinct from $Q_{1}$, we may select $\alpha$ in $Q_{1} \backslash Q_{2}$. Let $F=K(\alpha)$, and let $S=T \cap F$, the integral closure of $R$ in $F$. By (iv), $S$ is going-down, and hence treed. However, $Q_{1} \cap S$ and $Q_{2} \cap S$ are incomparable primes of $S$ which are both contained in $N \cap S$. This contradiction completes the proof.

Theorem 3.3 will settle Question 3.0 for cases in which, so to speak, 
the simple Galois groups arising from the proof of Proposition 3.1 are $Z / 2 Z$ and $2 / 3 Z$. First, we give a result of MCAdam [15], whose proof is reproduced here with his kind permission.

PROPOSITION 3.2 (McAdam). Let $R$ be a quasi-local integrally closed going-down ring. If $P$ is a prime ideal of $R$ and $b$ is an element of $R$, then $P$ and $R b$ are comparable.

Proof. Deny. Select $c$ in $P \backslash R b$, and set $u=c b^{-1}$. Observe that $R$ contains neither $u$ nor $u^{-1}$. Thus, if $M$ is the maximal ideal of $R$, [19, Theorem 7] implies that $N=M R[u]$ is a prime of $R[u]$ which contracts to $M$. Since $R \subset R[u]$ satisfies going-down, $N$ contains a prime $Q$ contracting to $P$. As $Q$ contains bu but not $b$, it follows that $u$ is in $Q$. Then $u$ is in $N$, so that $u$ satisfies a polynomial over $R$ with a unit coefficient. This contradicts the isomorphism $R[X] / M R[X] \rightarrow R[u] / N$ given in [19, Theorem 7$]$, to complete the proof.

THEOREM 3.3. The answer to Question 3.0 is affirmative if $L / K$ is algebraic and the group of K-algebra automorphisms of the normal closure of $L / K$ is finite of order $2^{e_{3} f}$ (e,f are non-negative integers).

proof. The problem may be reduced by reworking parts of the proof of Proposition 3.1. As in (iv), an application of Theorem 2.4 reduces to the case $L / K$ normal. As in (iii), replacing $K$ by the purely inseparable part of $L$, we may take $L / K$ finite Galois, with Galois group $G$ of order $2^{e} 3^{f}$. Now $G$ is solvable by Burnside's celebrated theorem [3, Theorem III], and so has a normal subgroup of index 2 or 3 . By working with the corresponding fixed field via Galois theory as in (ii), an induction on $|G|$ reduces to the case $|G|=2$ or 3 . If the theorem is denied for this case, we argue as in ( $v$ ) to obtain: incomparable primes $Q_{1}$ and $Q_{2}$ of $T$, both contained in a prime $N$ of $T$; primes $P=Q_{1} \cap R=Q_{2} \cap R$ and $M=N \cap R$ of $R$; and an element $\alpha$ in $Q_{1} \backslash Q_{2}$. As in (i), it is harmless to pass to $R_{M} \subset T_{R \backslash M}$, so that $R$ may be taken quasi-local with maximal ideal $M$.

$$
\text { 1: }|G|=2 \text {. Since } R \text { is integrally closed and }[L: K]=2 \text {, }
$$
it follows from [20, Chapter $\mathrm{V}$, Theorem 4] that there exist $a, b$ in $R$. such that 


$$
\alpha^{2}+a \alpha+b=0
$$

Note that $b$ is in $R \cap T \alpha \subset R \cap Q_{1}=P$. Consequently, $\alpha(\alpha+a)=-b$ is in $Q_{2}$, so that $\alpha+\alpha$ is in $Q_{2}$. Moreover, $a$ is not in $P$;

otherwise, $\alpha^{2}=-(a \alpha+b)$ would be in $P T \subset Q_{2}$, a contradiction. Thus $a^{2}$ is not in $P$, and an application of Proposition 3.2 shows that $P \subset R a^{2}$; in particular, $b a^{-2}$ is in $R$. Since

$$
\left(\alpha a^{-1}\right)^{2}+\alpha a^{-1}+b a^{-2}=0 \text {, }
$$

$\alpha a^{-1}$ lies in $T$. Now $Q_{1}$ contains $\left(\alpha a^{-1}\right) a$ but not $a$; so $\alpha a^{-1}$ is in $Q_{1}$. Furthermore, $Q_{2}$ contains $\left(\alpha a^{-1}+1\right) a$ but not $a ;$ so $\alpha a^{-1}+1$ is in $Q_{2}$. Thus $1=\left(\alpha a^{-1}+1\right)-\alpha a^{-1}$ is in $Q_{1}+Q_{2} \subset N$, the desired contradiction.

Case 2: $|G|=3$. As above, [20, Chapter V, Theorem 4] provides $a, b, c$ in $R$ such that

$$
\alpha^{3}+a \alpha^{2}+b \alpha+c=0
$$

By reasoning as above, $c$ is in $P$ and $\beta=\alpha^{2}+a \alpha+b$ is in $Q_{2}$.

We claim that we may assume that $b$ is in $P$. If not, $B$ is in $Q_{2} \backslash Q_{1}$. Moreover, one may laboriously check (see the remark following the proof) that $\beta$ satisfies

$$
\beta^{3}-b \beta^{2}+a c \beta-c^{2}=0
$$

As ac (and $-c^{2}$ ) are in $P$, the claim is sustained merely by replacing $\alpha$ by $\beta$.

Now that $b$ is in $P$, the argument proceeds as in Case 1 . Note that $a$ is not in $P$. Then observe by Proposition 3.2 that $b a^{-2}$ and $c a^{-3}$ are in $R$. Since

$$
\left(\alpha a^{-1}\right)^{3}+\left(\alpha a^{-1}\right)^{2}+\left(b a^{-2}\right)\left(\alpha a^{-1}\right)+c a^{-3}=0
$$


$\alpha a^{-1}$ is in $T$, and hence in $Q_{1}$. However $\alpha+a$ is in $Q_{2}$ (since $\alpha(\alpha+a)$ is). Thus $\alpha a^{-1}+1$ is in $Q_{2}$, and once again we have the desired contradiction: 1 in $N$, to complete the proof.

REMARK. The key to generalizing Theorem 3.3 to certain groups whose orders involve primes other than 2 and 3 may lie in suitably identifying the coefficients of minimum polynomials of elements constructed, like $\beta$, from the minimum polynomial of $\alpha$. The coefficients $-b, a c,-c^{2}$ of $\beta^{\prime} s$ polynomial were, of course, obtained with the aid of $\alpha$ 's polynomial by Cramer's rule from a system of three linear equations, whose coefficient matrix had (nonzero) determinant equal to $c$.

Besides Question 3.0, another ascent problem is to determine conditions on a going-down ring $R$ which guarantee that $\bar{R}$ is going-down. (The extension $D \subset V^{*}$ in Example 2.1 shows that the corresponding descent problem is complicated; see, however, Corollary 2.5.) In this regard, we next offer a result which generalizes [6, Proposition 2.7] and [8, Proposition 25.13]. As usual $h t$, dim, and $\underset{v}{\operatorname{dim}}$ will denote height, Krull dimension, and valuative dimension, respectively.

PROPOSITION 3.4. Let $R$ be going-down. Assume that all the maximal ideals of $R$ have the same finite height $n$. Let $T$ be an integrally closed domain which is integral over $R$. Then $T$ is Prüfer if and only if $\underset{v}{\operatorname{dim}}(R)=n$.

Proof. The "only if" half is immediate since $\underset{v}{\operatorname{dim}}(R)=\underset{v}{\operatorname{dim}}(T)$, by [8, Proposition 25.12].

Conversely, assume that $\operatorname{dim}(R)=n$ and that $T$ is not Prïfer. Choose a maximal ideal $N$ of $T$ such that $T_{N}$ is not a valuation ring; set $M=N \cap R$. By integrality, $M$ is maximal; so $h t(M)=n$.

Select $u$ in the quotient field of $T$ such that $T_{N}$ contains neither $u$ nor $u^{-1}$. By [19, Theorem 7$], Q=N T_{N}[u]$ is a nonmaximal 
prime of $T_{N}[u]$ contracting to $M$. Since $R \subset T_{N}[u]$ satisfies goingdown, we see that $h t(Q) \geq h t(M)=n$. Then

$$
n=\operatorname{dim}_{v}(T) \geq \operatorname{dim}\left(T_{N}[u]\right) \geq 1+h t(Q) \geq n+1,
$$

the desired contradiction.

Our final result is motivated by the observation that the rings $V$ and $D$ in Example 2.1 have valuative dimension 2 .

COROLLARY 3.5. Let $R$ be going-down, with $\operatorname{dim}(R)=2$, and let $T$ be an integrally closed domain which is integral over $R$. Then $T$ is going-down.

Proof. Let $Q \subset N$ be distinct primes of $T$, and let $V$ be an overring of $T$ with a prime contracting to $N$. Set $M=N \cap R$. To show that going-down obtains for the present data in $T \subset V$, we may suppose that $h t(M)=2$. (Otherwise, $Q=0$, a trivial case.) As it is enough to prove that $T_{R W M} \subset V_{R \backslash M}$ satisfies going-down, it surely suffices to show that $T_{R \backslash M}$ is Prüfer. This, however, follows from Proposition 3.4, as applied to $R_{M} \subset T_{R \backslash M}$, since

$$
2=h t(M)=\operatorname{dim}\left(R_{M}\right) \leq \operatorname{dim}_{v}\left(R_{M}\right) \leq \underset{v}{\operatorname{dim}}(R)=2 .
$$

We close by observing that Proposition 3.4 leads easily to an amusing proof of Prüfer's ascent result for the case that the given Prüfer domain contains no primes of infinite height.

\section{References}

[1] Charles H. Brase, "A note on intersections of valuation ideals", Proc. Amer. Math. Soc. 38 (1973), 37-39.

[2] J.W. Brewer and W.J. Heinzer, "Ideals $I$ of $R[X]$ for which $R[X] / I$ is R-projective", Proc. Amer. Math. Soc. 43 (1974), 21-25.

[3] W. Burnside, "On groups of order $p^{\alpha} q^{\beta}$ ", Proc. London Math. Soc. (2) 1 (1904), 388-392. 
[4] Jeffrey Dawson and David E. Dobbs, "On going down in polynomial rings", Canad. J. Math. 26 (1974), 177-184.

[5] David E. Dobbs, "On going down for simple overrings", Proc. Amer. Math. Soc. 39 (1973), 515-519.

[6] David E. Dobbs, "On going down for simple overrings II", Comm. Algebra 1 (1974), 439-458.

[7] David E. Dobbs and Ira J. Papick, "On going-down for simple overrings. III", Proc. Amer. Math. Soc. 54 (1976), 35-38.

[8] Robert W. Gilmer, Multiplicative ideal theory, Part I (Queen's Papers on Pure and Applied Mathematics, 12. Queen's University, Kingston, Ontario, 1968).

[9] William J. Heinzer, "Some properties of integral closure", Proc. Amer. Math. Soc. 18 (1967), 749-753.

[10] William Heinzer and Jack Ohm, "The finiteness of $I$ when $R[X] / I$ is R-flat. II", Proc. Amer. Math. Soc. 35 (1972), 1-8.

[11] Irving Kaplansky, Commutative rings (Allyn and Bacon, Boston, 1970).

[12] Wolfgang Krull, "Beiträge zur Arithmetik kommutativer

Integritätsbereiche. III: Zum Dimensionsbegriff der Idealtheorie", Math. 2. 42 (1936-1937), 745-766.

[13] William J. Lewis, "The spectrum of a ring as a partially ordered set", J. AZgebra 25 (1973), 419-434.

[14] Stephen McAdam, "Going down", Duke Math. J. 39 (1972), 633-636.

[15] Stephen McAdam, Private communication, May 1974.

[16] Ira J. Papick, "Topologically defined classes of going-down rings", Trans. Amer. Math. Soc. 219 (1976), 1-37.

[17] Heinz Prüfer, "Untersuchungen über Teilbarkeitseigenschaften in Körpern", J. reine angew. Math. 168 (1932), 1-36.

[18] Fred Richman, "Generalized quotient rings", Proc. Amer. Math. Soc. 16 (1965), 794-799.

[19] A. Seidenberg, "A note on the dimension theory of rings", Pacific $J$. Math. 3 (1953), 505-512. 
[20] Oscar Zariski and Pierre Samuel, Commutative algebra, Volume I (Van Nostrand, Princeton, New Jersey; Toronto; New York; London; 1958). See also: Oscar Zariski, Pierre Samuel, Commutative algebra, Volume 1 (Graduate Texts in Mathematics, 28. SpringerVerlag, New York, Heidelberg, Berlin, 1975).

Department of Mathematics,

University of Tennessee,

Knoxville,

Tennessee, USA. 